\title{
Density of states and magnetoconductance of disordered Au point contacts
}

\author{
L.H. Yu and D. Natelson \\ Department of Physics and Astronomy, Rice University, 6100 Main St., Houston, TX 77005
}

(Dated: November 19, 2018)

\begin{abstract}
We report the first low temperature magnetotransport measurements on electrochemically fabricated atomic scale gold nanojunctions. As $T \rightarrow 0$, the junctions exhibit nonperturbatively large zero bias anomalies (ZBAs) in their differential conductance. We consider several explanations and find that the ZBAs are consistent with a reduced local density of states (LDOS) in the disordered metal. We suggest that this is a result of Coulomb interactions in a granular metal with moderate intergrain coupling. Magnetoconductance of atomic scale junctions also differs significantly from that of less geometrically constrained devices, and supports this explanation.
\end{abstract}

PACS numbers:

At low temperatures disordered metals exhibit a reduced local density of states (LDOS) at the Fermi level, seen as a zero bias anomaly (ZBA) in tunneling spectroscopy (e.g. [1]). This is the result of disorder-enhanced electron-electron interactions [2]. Granular metals with large intergrain conductances $(g \equiv$ $\left.\left\langle G_{i, j}\right\rangle /\left(2 e^{2} / h\right)>>1\right)$ are predicted[3] to have LDOS suppressions approaching the perturbative result 2] for weakly disordered films. With strong disorder and geometric constraint [4, 5], the ZBA in metal films can approach $100 \%$, ascribed to a correlation gap due to strong Coulomb interactions [6]. Similarly, weakly coupled granular metals $(g<<1)$ should act as arrays of tunnel junctions, with an exponentially suppressed tunneling LDOS [3] as $T \rightarrow 0$.

Metallic nanojunctions (MNJs) are tools to examine geometrically constrained, disordered metals on the nanometer scale. While clean break junctions made in ultrahigh vacuum (UHV) have been studied extensively []], nanojunctions between highly disordered metals are comparatively unexplored.

We present the first low temperature measurements of atomic scale metal junctions made by electrochemical deposition, a method proposed for molecular electronics investigations 8,9$]$. Such metals may be disordered by grain boundaries, ionic impurities, and surface adsorbates. As we reported elsewhere 10], in "large" junctions $\left(G(300 \mathrm{~K})>>G_{0} \equiv 2 e^{2} / h\right)$, small ZBAs are consistent with the perturbative theory of Altshuler, Aronov and Lee (AAL) [2]. When $G(300 K) \sim 2 e^{2} / h$, however, low $T$ conductance suppression approaches $100 \%$. Here we consider several models and show that these junctions are atomic scale probes of the LDOS of the disordered metal leads, which exhibit non-perturbative, temperature-dependent LDOS corrections. We give a phenomenological description of the LDOS suppression, and argue that its likely origin is the granular character of the electrodeposited material. Finally, these junctions exhibit nontrivial weak localization magnetoconductances, consistent with junction size and the hypothesis of granularity.

The MNJs are prepared by electrochemistry starting from electrodes defined by e-beam lithography. The de-

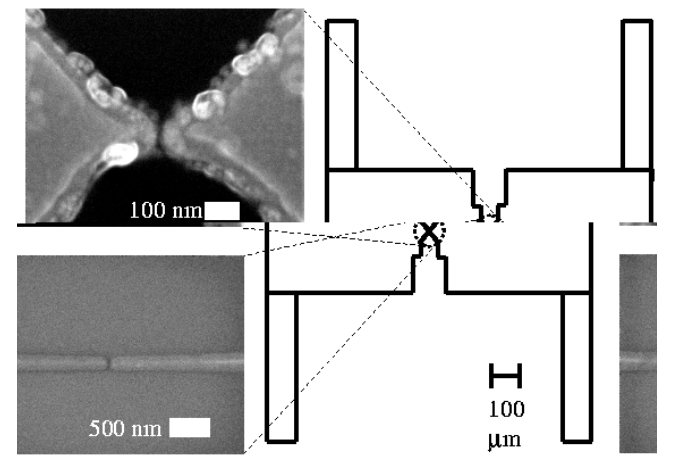

FIG. 1: Schematic of the electrode configurations used to make the nanoscale junctions. At right, overall electrode geometry; at upper left, pre-deposition image of tip geometry used for sample C; lower left, pre-deposition image of tip geometry used for samples A and B. Samples A, B, and C are representative atomic scale junctions, as discussed below.

tails of sample fabrication are available elsewhere 10. Gold electrodes $25 \mathrm{~nm}$ thick with separations of $\sim 20 \mathrm{~nm}$ are prepared on $p+$ silicon substrates coated with $200 \mathrm{~nm}$ of thermal oxide. Electrode configurations are shown in Fig. [1 Lumped capacitance between each electrode and the substrate is estimated to be $\sim 50 \mathrm{pF}$. The electrodes are covered by $20 \mathrm{~nm} \mathrm{Al}{ }_{2} \mathrm{O}_{3}$ during evaporation, limiting electrochemistry to the electrode edges. The evaporated $\mathrm{Au}$ has a typical resistivity of $5 \mu \Omega$-cm.

Additional gold is deposited using a buffered aqueous solution of potassium cyanaurate [8], while interelectrode conductance is monitored with standard lock-in techniques. Discrete conductance steps on the order of $G_{0}$ are observed during junction formation, corresponding to atomic reconfigurations. The MNJ is grown to a specified conductance, rinsed in deionized water, and dried with dry nitrogen. Through measurements on test structures, we find that the average resistivity of the electrodeposited gold is $\sim 35 \mu \Omega$-cm at $4.2 \mathrm{~K}$, corresponding to an elastic mean free path of $\sim 2.5 \mathrm{~nm}$, much shorter than that of the evaporated $\mathrm{Au}$.

Spontaneous conductance switching and its strong suppression as $T$ is lowered suggest that the junctions consist of a small number of atoms that can diffuse readily at room temperature. Surviving junctions with $G \sim$ 


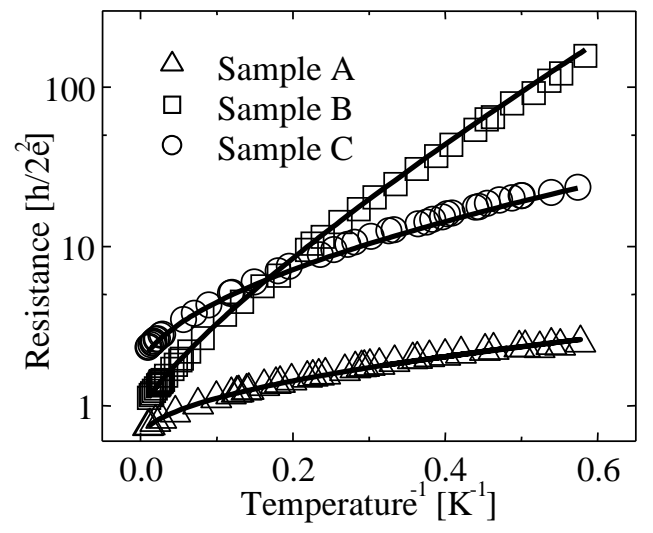

FIG. 2: Zero bias resistance as a function of temperature for three samples with room temperature conductances on the order of $G_{0}$. The form of the dependence is nonactivated. Solid lines are fits: (A) $0.61 \exp \left(1.91 T^{-0.5}\right)$; (B) $0.91 \exp \left(8.06 T^{-0.8}\right) ;$ (C) $1.65 \exp \left(3.61 T^{-0.56}\right)$.

$1-10 G_{0}$ are stable for tens of minutes, typical of junctions not prepared in UHV[11].

We have successfully measured ten nanojunctions with room temperature conductances ranging from 0.5 to 200 $G_{0}$ in a variable temperature cryostat. Using standard quasi-4-terminal lock-in techniques we have measured nanojunction differential conductance $G(V, T)=d I / d V$ (and differental resistance) as a function of temperature, dc bias voltage, gate voltage, and magnetic field. All nanojunctions are Ohmic up to $200 \mathrm{mV}$ at $300 \mathrm{~K}$.

As discussed elsewhere 10], high conductance junctions (e.g. $\left.G(300 \mathrm{~K}) \sim 30 G_{0}\right)$ typically exhibit a small $(15 \%$ at $1.8 \mathrm{~K}) \mathrm{ZBA}$, logarithmic in temperature below $30 \mathrm{~K}$. We interpret this ZBA and its scaling with bias voltage as consistent with the AAL perturbative DOS suppression. An analysis of the Coulomb interaction with a single coherent scatterer 13] can quantitatively explain this data 14], provided the disordered metal is granular, with an intergrain conductance on the order of $50 G_{0}$; such an analysis is only applicable for systems with $G>>G_{0}$.

In atomic scale junctions prepared as above with $G(300 K) \sim 1 G_{0}$, we find ZBAs approaching $100 \%$ conductance suppression as $T \rightarrow 0$. Such deep ZBAs are never seen in the larger junctions and are present in both differential conductance and resistance measurements. The only difference between large and atomic scale junctions is a brief amount of electrodeposition time; the microstructure of the deposited material is identical. Figure 2 shows the zero bias resistance vs. $T$ on Arrhenius-style axes for three of these samples.

We now present evidence that these large ZBAs are caused by nonperturbative low temperature corrections to the LDOS of the electrodeposited material. Figure 3 (left inset) shows $G(V=0, T)$ for sample A $\left(\mathrm{G}(\mathrm{T}=300 \mathrm{~K})=1 G_{0}\right)$ as a function of temperature cycling. During initial cooling (trace 1), $G$ varies little until $T<50 \mathrm{~K}$, when the zero bias suppression begins. At $15 \mathrm{~K}, G$ spontaneously increases by about $0.6 G_{0}$. The conductance then continues to decrease upon cool-

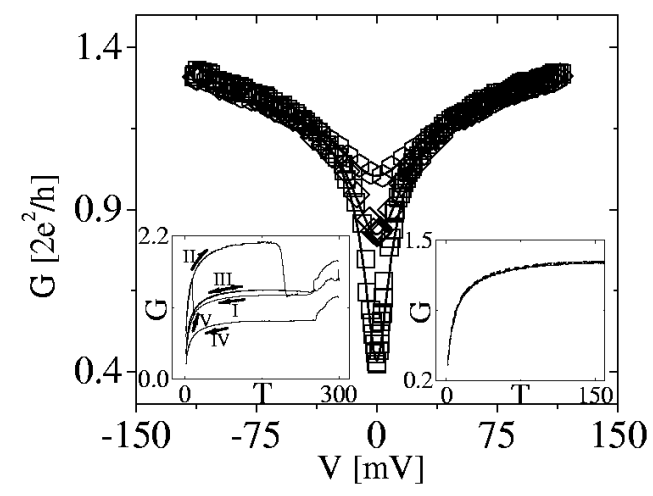

FIG. 3: Zero bias anomaly for Sample A at (top to bottom) $20 \mathrm{~K}, 10 \mathrm{~K}$, and $2 \mathrm{~K}$. Solid lines are the phenomenological model described in text. Left inset: various branches of $G(0, T)$ for this sample, each branch corresponding to a different arrangement of contact atoms. Right inset: all the branches collapse onto a single curve when each is scaled by a multiplicative constant.

ing. When the nanojunction is warmed (trace 2), the high temperature conductance appears to be $\sim 2 G_{0}$. At $220 \mathrm{~K}$, however, $G$ spontaneously decreases by about 0.85 $G_{0}$, returning near its original value. Repeated thermal cycling and LED illumination at $2 \mathrm{~K}$ result in other branches of $G$ vs. $T$ (traces 3,4,5). All these $G(V=0, T)$ curves collapse onto one curve (right inset) when each branch of $G(T)$ is multiplied by a non-integer constant.

The discrete changes in $G$ strongly support the idea that this junction's low room temperature conductance $\left(\sim G_{0}\right)$ is due to the junction's atomic scale. The addition of a single partially transmitting channel upon cooling occurs as thermal contraction slightly decreases the interelectrode distance. Thermal expansion on warming stretches the junction, and the additional channel is lost, just as in hysteresis seen in mechanical break junction measurements 15. These serial rearrangements indicate that this junction was in a fortuitous regime of stability. In the other samples, when rearrangements took place the junctions either broke completely or coalesced to a high conductance state.

For each conductance branch, the ZBA is measured at several temperatures. Figure 3 shows the conductance versus bias voltage at $2 \mathrm{~K}, 10 \mathrm{~K}$, and $20 \mathrm{~K}$ for this nanojunction in one of its configurations. The same factors used to collapse the $G(V=0, T)$ branches also collapse the bias sweep data onto a single set of curves. It is clear that $G(V, T)$ is only multiplicatively scaled by discrete atomic rearrangements of the small number of conducting channels.

We now consider possible origins of such a nonperturbative ZBA. A successful explanation must be consistent with: (a) the temperature dependence of the zero bias conductance; (b) the functional form of the ZBA vs. bias voltage and temperature; and (c) the scaling of the ZBA data with junction conductance as shown in Fig. 3.

Coulomb blockade in an array of grains weakly coupled by tunnel junctions can produce a ZBA [16]. However, the 


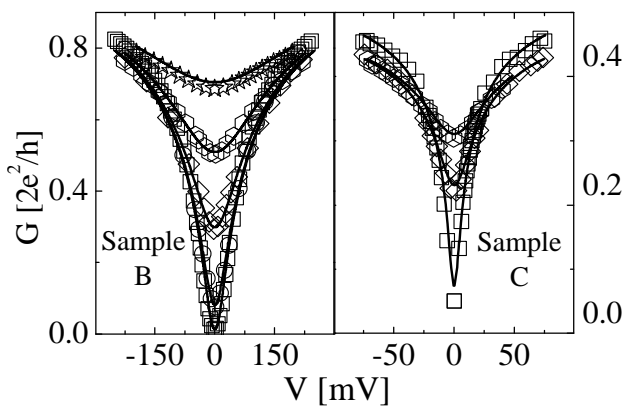

FIG. 4: Bias sweep data for two other low conductance junctions. Temperatures for sample B (left) are, top to bottom, $40 \mathrm{~K}, 20 \mathrm{~K}, 10 \mathrm{~K}, 4 \mathrm{~K}$, and $2 \mathrm{~K}$. Temperatures for sample C (right) are, top to bottom, $20 \mathrm{~K}, 10 \mathrm{~K}$, and $2 \mathrm{~K}$. Solid lines are calculated using the phenomenological LDOS described in the text.

temperature dependence in Fig. 2 2 and the ZBA width of our data are incompatible with such a simple model. For a small number of metal islands, one would also expect asymmetries or Coulomb staircase effects, neither of which are observed. Gate voltage sweeps over a $100 \mathrm{~V}$ range produce no measurable response at any temperature considered. Finally, this picture would not explain the scaling in Fig. 3

Another candidate is environmental Coulomb blockade (ECB) 17]. Conduction through a single junction in series with an environmental impedance is suppressed at low $T$ and $V$ because tunneling electrons excite electromagnetic modes of the environment, reducing the phase space for tunneling 18]. The $I V$ characteristics are determined by $P(E)$, the probability of a tunneling electron to excite an environmental mode of energy $E$. The form of $P(E)$ depends on the impedance of the environment, which typically must be well controlled to observe ECB in single junctions.

ECB has been seen [19] in clean point contacts made using mechanical break junctions. The environmental impedance was modeled as the junction capacitance in parallel with the lead resistance 19]. Our voltage and temperature scales would imply a charging energy $10^{2} \times$ that in Ref. [19]. This seems unlikely given our similar geometry and greater stray capacitance. Furthermore, in atomic scale junctions the ECB suppression scales with a Fano factor under changes of channel transmittance [20], contradicting Fig. 3. This Fano scaling is expected to hold for a general environmental impedance [21], seemingly ruling out ECB as the origin of the observed ZBAs.

A tunneling model[2] is a natural approach to analyzing the ZBA data. One can consider a single tunnel junction with a transmission probability $T(E)$ and an effective $\operatorname{LDOS} \nu(E, T)$. The conductance $G$ normalized by the high bias / high temperature conductance $G_{h}$ is then proportional to $T(E) \nu(E, T) / \nu_{0}$ convolved with a thermal spreading function. Here $\nu_{0}$ is the background effective density of states. Our ZBA data exhibit a considerably stronger $T$-dependence than the spreading function; therefore a $T$-dependent LDOS is required in this model.
In this picture the transmittance is independent of $T$ and (slowly varying in) $E$, but depends on the precise configuration of the few atoms that make up the junction. The LDOS is determined by disorder "built in" during the electrodeposition process. This would naturally explain the scaling in Fig. 3. different branches are equivalent to probing the same LDOS suppression using tunnel junctions of various sizes.

The large ZBAs in the atomic scale junctions, reminiscent of correlation gaps in highly disordered metal films [4, 5], imply a nonperturbative LDOS suppression. Efetov and Tscherich [3] consider the LDOS in granular metals having a dimensionless intergrain conductance $g$. They derive expressions for the LDOS in the limit $g>>1$ $(g<<1)$, but the calculated $T$-dependence is too gradual (steep) to fit our data. This suggests that our samples fall between these extremes, into the intermediate range of $g$ for which there is currently no analytic expression.

A generalized treatment of corrections to electron tunneling 22] accounts nonperturbatively for both intraelectrode Coulomb effects (the AAL LDOS correction) and interelectrode Coulomb interactions in the presence of an electromagnetic environment $(P(E)$ theory). Effective tunneling densities of states have been calculated that agree well with experiments on spatially extended tunnel junctions 23]. One would expect some form of this generalized theory to apply in the granular metal case. We introduce an ansatz for the functional form of $\delta \nu(\epsilon, T)$ :

$$
\delta \nu(\epsilon, T)=\nu_{0}\left(1-\operatorname{erf} \sqrt{\frac{-e \Gamma}{\sqrt{\epsilon^{2}+(a+b T)^{2}}}}\right)
$$

where $\Gamma, a$, and $b$ are sample-specific, temperature independent parameters. This model LDOS is able to describe empirically the ZBA data over a broad temperature and voltage range. The $T=0$ version of this form is derived 22 for $1 \mathrm{~d}$ tunnel junctions, and is also equivalent to ECB in an ultrasmall junction connected to an $R C$ transmission line 18, 22]. However, the Fano factors discussed above for ECB in point contacts make this interpretation difficult to reconcile with the observed scaling. The solid lines in Figs. 3 and 4 are fits using Eqn. (11). The relevant parameters is shown in Table 【

\begin{tabular}{|c|c|c|c|c|}
\hline Sample & $G_{h}\left[2 e^{2} / h\right]$ & $e \Gamma[\mathrm{J}]$ & $a[\mathrm{~J}]$ & $b[\mathrm{~J} / \mathrm{K}]$ \\
\hline $\mathrm{A}$ & $1.51^{*}$ & $2.96 \times 10^{-22}$ & $2.33 \times 10^{-22}$ & $1.43 \times 10^{-22}$ \\
$\mathrm{~B}$ & 1.29 & $5.11 \times 10^{-21}$ & $1.70 \times 10^{-22}$ & $6.94 \times 10^{-22}$ \\
$\mathrm{C}$ & $0.57^{* *}$ & $6.07 \times 10^{-22}$ & $1.87 \times 10^{-23}$ & $1.57 \times 10^{-22}$ \\
\hline
\end{tabular}

TABLE I: Model parameters from Eq. 1) used to reproduce the ZBA data for the samples shown in Fig. 2 *For sample A, data from branch number 3 from Fig. 3 were used for the fits. ${ }^{*}$ For sample $\mathrm{C}$, a slight junction rearrangement led us to use $G_{h}=0.62$ to fit data at $2 \mathrm{~K}$, with the other parameters unchanged.

We also observe magnetoconductance (MC) in atomic scale junctions that differs from that in larger, cleaner 


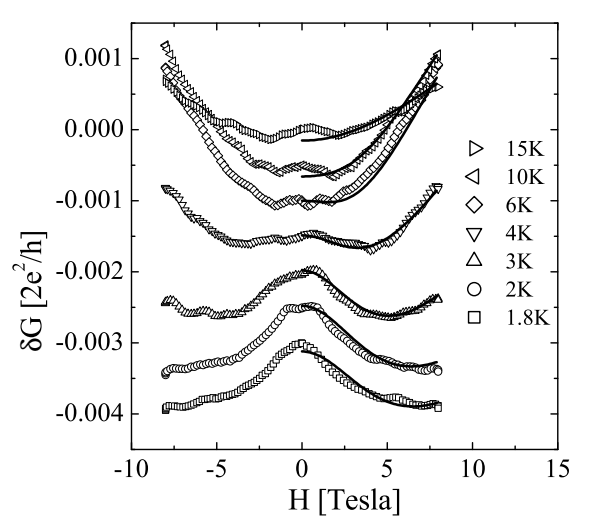

FIG. 5: Weak localization magnetoconductance sweeps at several temperatures for sample B, the junction from Fig. 4 Curves have been offset vertically for clarity.

gold systems. Quantum MC effects are dominated by the coherent volume centered on the conductance-limiting junction. Larger junctions and lead electrodes exhibit weak antilocalization similar to that observed in clean gold films, because the spin-orbit scattering time, $\tau_{\mathrm{SO}}$, is more rapid than the inelastic time $\tau_{\phi}[24]$. While $\tau_{\mathrm{SO}}$ is temperature independent, $\tau_{\phi}$ typically increases with decreasing $T$, and $\tau_{\phi}>\tau_{\mathrm{SO}}$ for evaporated Au films below $20 \mathrm{~K}$.

Figure 5 shows MC data for Sample B (data for other atomic scale samples are similar). Curves are offset by $0.0005 G_{0}$. Positive MC contributions suggest that $\tau_{\phi}$ is comparable with $\tau_{\mathrm{SO}}$, with apparently $\tau_{\mathrm{SO}}>\tau_{\phi}$ for $T>\sim 6 \mathrm{~K}$.

Quantitative analysis is difficult because the coherent volume around the constriction is of uncertain dimensionality with respect to diffusion and quantum coherence. As a qualitative guide, solid curves are fits using $1 \mathrm{~d}$ weak localization, assuming $3 \mathrm{~d}$ diffusion, an effective width of $3 \mathrm{~nm}$ (roughly sets the field scale) and an effective length of 5 microns (essentially a fixed numerical factor to scale the conductance axis), and allowing $L_{\phi}$ and $L_{\mathrm{SO}}$ to vary at each temperature. A natural explanation for a varying $L_{\mathrm{SO}}$ would be a fixed $\tau_{\mathrm{SO}}$ but a temperature dependent diffusion constant, $D(T)$, that decreases as $T \rightarrow 0$. Such a dependence supports the granular metal model, where $D(T)$ would vary as intergrain interactions become important with decreasing $T$. We find that the ratio $\left(L_{\phi} / L_{\mathrm{SO}}\right)^{2}=\tau_{\phi}(T) / \tau_{\mathrm{SO}}$ (independent of $D$ ) increases with decreasing $T$ roughly like $1 / T$. The fact that $\tau_{\phi} / \tau_{\mathrm{SO}} \sim 1$ here suggests that $\tau_{\phi}$ is shorter here than in larger junctions.

These first low- $T$ studies of electrochemically made atomic scale $\mathrm{Au}$ nanojunctions have revealed significant departures from the properties seen in larger and cleaner $\mathrm{Au}$ structures. Atomic scale junctions locally probe a suppressed density of states in the disordered leads. We provide an expression that describes the data pheonomenologically, and suggest an underlying physical origin for the effect consistent with magnetoconductance data. Further studies are required to determine conclusively the physics behind the gap observed in the LDOS and the nontrivial MC properties of this system when examined at the atomic scale.

The authors gratefully acknowledge the support of the Robert A. Welch Foundation and the Research Corporation.
[1] R.C. Dynes and J.P. Garno, Phys. Rev. Lett. 46, 137 (1981).

[2] B. L. Altshuler and A. G. Aronov, in Electron-Electron Interactions in Disordered Systems, A. L. Efros and M. Pollak, eds. (North Holland, New York, 1985) 1.

[3] K.B. Efetov and A. Tscherich, preprint: cond-mat/0302257 (2003).

[4] V. Yu. Butko, J.F. DiTusa, and P.W. Adams, Phys. Rev. Lett. 84, 1543 (2000).

[5] E. Bielejec, J. Ruan, and Wenhao Wu, Phys. Rev. Lett. 87, 036801 (2001).

[6] A.L. Efros and B.I. Shklovskii, J. Phys. C 8, L49 (1975).

[7] J.M. van Ruitenbeek, in Metal Clusters on Surfaces: Structure, Quantum Properties, Physical Chemistry, K.H.Meiwes-Broer, ed. (Springer-Verlag, Berlin, 2000); H.E. van den Brom and J.M. van Ruitenbeek, Phys. Rev. Lett. 82, 1526 (1999); B. Ludoph, M.H. Devoret, D. Esteve, C. Urbina, and J.M. van Ruitenbeek, Phys. Rev. Lett. 82, 1530 (1999).

[8] A.F. Morpurgo, C.M. Marcus, and D.B. Robinson. App. Phys. Lett. 74 (1999) 2084.

[9] C.Z. Li, H.X. He, A. Bogozi, J.S. Bunch, and N.J. Tao.
App. Phys. Lett. 76 (2000) 1333; S. Boussaad and N. J. Tao. Appl. Phys. Lett. 80 (2002) 2398.

[10] L.H. Yu and D. Natelson. Appl. Phys. Lett. 82, 2332 (2003).

[11] K. Hansen, S. K. Nielsen, M. Brandbyge, E. Laegsgaard, I. Stensgaard, and F. Besenbacher. Appl. Phys. Lett. $7 \mathbf{7}$ (2000) 708 .

[12] H. B. Weber, R. Häussler, H. v. Löhneysen, and J. Kroha. Phys. Rev. B 63 (2001) 165426.

[13] D.S. Golubev and A.D. Zaikin. Phys. Rev. Lett. 86, 4887 (2001).

[14] A.D. Zaikin, private communication.

[15] H.E. van den Brom, A.I. Yanson, and J.M. van Ruitenbeek. Physica B 252, 69 (1998).

[16] J.P. Pekola, L.J. Taskinen, and Sh. Farhangfar. Appl. Phys. Lett. 76, 3747 (2000).

[17] P. Delsing, K.K. Likharev, L.S. Kuzmin, and T. Claeson. Phys. Rev. Lett. 63, 1180 (1989); M.H. Devoret, D. Esteve, H. Grabert, G.-L. Ingold, H. Pothier, and C. Urbina. Phys. Rev. Lett. 64, 1824 (1990).

[18] Single Charge Tunneling, edited by H. Grabert and M. Devoret, NATO ASI, Ser. B, Vol. 294 (Plenum, New 
York, 1992).

[19] R. Cron et al., in Electronic Correlations: From Mesoto Nanophysics, T. Martin, G. Montambaux, and J. Tran Thanh Van eds., EDPSciences (2001) 17.

[20] A.L. Yeyati, A. Martin-Rodero, D. Esteve, and C. Urbina. Phys. Rev. Lett. 87, 046802 (2001).

[21] R. Cron, Ph.D. thesis (2001).
[22] J. Rollbühler and H. Grabert, Phys. Rev. Lett. 87, 126804 (2001).

[23] F. Pierre, H. Pothier, P. Joyez, N.O. Birge, D. Esteve, and M.H. Devoret, Phys. Rev. Lett. 86, 1590 (2001).

[24] G. Bergmann. Z.Phys. B 48, 5 (1982). 